Article

\title{
Bödewadt Flow Over a Permeable Disk with Homogeneous-Heterogeneous Reactions: A Numerical Study
}

\author{
Talat Rafiq ${ }^{\circledR}$ and Meraj Mustafa Hashmi * \\ School of Natural Sciences (SNS), National University of Sciences and Technology (NUST), Islamabad 44000, \\ Pakistan; talatrafique2@gmail.com \\ * Correspondence: meraj_mm@hotmail.com; Tel.: +92-51-90855585
}

Received: 10 July 2019; Accepted: 15 August 2019; Published: 27 September 2019

check for updates

\begin{abstract}
We analyzed the onset of homogeneous-heterogeneous reactions in Bödewadt flow occurring over an isothermal and permeable surface. This research is based on the assumption that the homogeneous (bulk) reaction follows isothermal cubic autocatalator kinetics, whereas the surface reaction is governed by first-order kinetics. The heat energy released during the chemical reaction is assumed to be negligible. The governing equations are reducible to a set of self-similar equations, which are handled numerically. Asymptotic analysis was conducted, which revealed that the existence of a concentration boundary layer on the disk is possible only when the disk is subjected to a sufficient amount of suction. In a large suction situation, an exact formula for concentration profile $\phi$ was derived that strongly supports the obtained numerical solution. Our results demonstrate the mass transfer parameter considerably alters flow fields. The concentration at the wall varies substantially when the chemical reaction proceeds at a faster rate.
\end{abstract}

Keywords: asymptotic analysis; chemical reaction; porous boundary; Bödewadt flow; self-similar solution

\section{Introduction}

Rotating flows have been proven stimulating since they are predominant in a number of industrial products and processes such as computer disk drives, chemical mixing chambers, viscometers, car breaking systems, electrochemistry, and food processing technologies. The thermal transport in these rotating-disk systems has been a research focus for years. The steadily revolving flow occurring above an infinite plane (through the origin), rotating with some fixed angular velocity about the vertical axis, is one of the few problems for which an explicit solution of the Navier-Stokes equations can be sought. This also explains why it has received repeated attention. This problem was discovered and resolved by von-Kármán [1] using a self-similar transformation. Its cousin flow problem, which involves the steady rotation of viscous fluid high above a stationary infinite plane, was discovered by Bödewadt [2] in 1940. In Bödewadt's flow, fluid particles surrounding the disk move inward toward the axis of rotation under radial pressure gradient that is induced outside the boundary layer where fluid is in a state of rigid-body rotation. For the flow field to remain mass-conserving, an upward axial flow is established. This kind of flow is apparent in a tea cup where fluid rotation is induced by stirring and again after flow is left to itself for a short while. Bödewadt flow is experienced in other processes such as flow established on the stator of an enclosed rotor-stator system and chemical mixing chambers. Bödewadt's solution approach involved a power-series expansion about $z=0$ and an asymptotic expansion valid high above the boundary. Initial attempts in this area include the works of Batchelor [3], Rogers and Lance [4], Ackroyd [5], Watson and Wang [6] and Fransaer et al. [7]. More recently, researchers have shown an increasing interest in exploring Bödewadt flow. For example, 
the thermal transport in Bödewadt slip flow was numerically investigated by Sahoo [8]. Sahoo et al. [9] later applied two different numerical approaches to compute Bödewadt flow of Reiner-Rivlin fluid. Turkyilmazoglu [10] discussed boundary layer development in Bödewadt flow when the stationary disk was allowed to expand circumferentially. This flow model was revisited by Mustafa et al. [11] for water-based nanofluids using the Keller-Box procedure. Rahman and Andersson [12] considered heat transfer in pure Bödewadt flow and found that a physically compatible solution is achievable only when the disk is subjected to an adequate amount of suction. Bödewadt flow over a stretching disk in a nanofluid was formulated by Khan et al. [13] through a model that allows for passively-controlled nanoparticle concentration at the disk. Motivated by previous work [12], Mustafa et al. [14] introduced a non-linear radiation aspect to the Bödewadt slip flow in the presence of wall suction.

Chemical reactions for which reactants and products are in the same phase are termed homogeneous reactions, whereas heterogeneous reactions can have reactants from two or more phases. Reactions occurring on a surface of a catalyst are heterogeneous reactions. Notable applications of such reactions can be found in the production of ceramics, hydrometallurgical trade, production of fog, food process, combustion of coal particles in fluidized beds, and many others. Chaudhary and Merkin [15] introduced a simple model of homogeneous-heterogeneous reactions in stagnation-point flow around a flat plate by assuming the same diffusion coefficients for the reactants and autocatalyst. They investigated the occurrence of multiple solutions and bifurcation for the developed flow model. Merkin [16] used the same model to analyze mass transfer process in the Blasius boundary layer. He realized that the surface reaction is a dominant mechanism near the leading edge. A similar model was adopted by Bachok et al. [17] to observe fluid flow around a stagnation point on a stretching or shrinking surface with chemical reaction. The model introduced in Chaudhry and Merkin [15] was again used by Khan and Pop [18] to examine self-similar flow of Walters' B fluid subject to chemical reaction. Hayat et al. [19] considered a chemical reaction model for viscoelastic fluid flow along a bi-directional stretching sheet. Hayat et al. [20] analyzed the homogeneous-heterogeneous reaction model for nanofluid flow near a rotating disk with non-uniform thickness. Maqsood et al. [21] investigated a self-similar system governing Maxwell fluid flow in a rotating system influenced by chemically reactive species. Several researchers explored the influence of reacting species in different boundary layer flows ([22-26] and references therein).

To the best of our knowledge, Bödewadt flow containing chemically reactive substances has not been discussed previously. To fill this gap, we implemented the novel homogeneous-heterogeneous reaction model [15] for Bödewadt flow near a porous surface. We developed a closed-form analytical formula in a large wall suction case. Our asymptotic analysis revealed that a wall permeability assumption is required for the concentration equation to have a physically compatible solution. Section 2 presents the governing self-similar system based on the usual von-Karman substitutions. In Section 3, the numerical results are derived addressing the role of chemical reaction on the pure Bödewadt flow. The key findings of this research are provided in the final section.

\section{Problem Statement}

Suppose that a viscous fluid revolves about the vertical axis with uniform angular velocity $\omega$, sufficiently high above a stationary surface aligned with the plane $z=0$. The considered surface is permeable with $V$ representing wall transpiration velocity. In line with [2], flow field is assumed to be steady, laminar, and incompressible, and it comprises chemically reacting species. Because the problem exhibits cylindrical symmetry, we work in cylindrical coordinates $(r, \varphi, z)$, with the corresponding velocity components being denoted by $\left(V_{r}, V_{\varphi}, V_{z}\right)$. We consider a cubic autocatalytic reaction [15]:

$$
\alpha+2 \beta \rightarrow 3 \beta ; \text { rate }=k_{c} a b^{2},
$$


whereas a single, isothermal, and first-order reaction occurs at the catalyst surface:

$$
\alpha \rightarrow \beta ; \text { rate }=k_{s} a
$$

where $a$ and $b$ represent the concentrations of chemical species $\alpha$ and $\beta$, respectively; and $k_{c}$ and $k_{s}$ are constants.

Given the above assumptions, the constitutive relationships result in the following equations:

$$
\begin{gathered}
\frac{\partial V_{r}}{\partial r}+\frac{V_{r}}{r}+\frac{\partial V_{z}}{\partial z}=0 \\
\rho\left(V_{r} \frac{\partial V_{r}}{\partial r}-\frac{V_{\varphi}^{2}}{r}+V_{z} \frac{\partial V_{r}}{\partial z}\right)=-\frac{\partial p}{\partial r}+\mu\left(\frac{\partial^{2} V_{r}}{\partial r^{2}}+\frac{\partial}{\partial r}\left(\frac{V_{r}}{r}\right)+\frac{\partial^{2} V_{r}}{\partial z^{2}}\right), \\
\rho\left(V_{r} \frac{\partial V_{\varphi}}{\partial r}+\frac{V_{r} V_{\varphi}}{r}+V_{z} \frac{\partial V_{\varphi}}{\partial z}\right)=\mu\left(\frac{\partial^{2} V_{\varphi}}{\partial r^{2}}+\frac{\partial}{\partial r}\left(\frac{V_{\varphi}}{r}\right)+\frac{\partial^{2} V_{\varphi}}{\partial z^{2}}\right), \\
\rho\left(V_{r} \frac{\partial V_{z}}{\partial r}+V_{z} \frac{\partial V_{z}}{\partial z}\right)=-\frac{\partial p}{\partial z}+\mu\left(\frac{\partial^{2} V_{z}}{\partial r^{2}}+\frac{1}{r} \frac{\partial V_{z}}{\partial r}+\frac{\partial^{2} V_{z}}{\partial z^{2}}\right), \\
V_{r} \frac{\partial a}{\partial r}+V_{z} \frac{\partial a}{\partial z}=D_{\alpha}\left(\frac{\partial^{2} a}{\partial r^{2}}+\frac{1}{r} \frac{\partial a}{\partial r}+\frac{\partial^{2} a}{\partial z^{2}}\right)-k_{c} a b^{2}, \\
V_{r} \frac{\partial b}{\partial r}+V_{z} \frac{\partial b}{\partial z}=D_{\beta}\left(\frac{\partial^{2} b}{\partial r^{2}}+\frac{1}{r} \frac{\partial b}{\partial r}+\frac{\partial^{2} b}{\partial z^{2}}\right)+k_{c} a b^{2} .
\end{gathered}
$$

where $\rho$ denotes the fluid density, $\mu$ is the dynamic viscosity, $D_{\alpha}$ and $D_{\beta}$ are the respective diffusion coefficients, and $p$ represents pressure. Outside the boundary layer, the centrifugal force is balanced by radial pressure gradient. Mathematically,

$$
\frac{\partial p}{\partial r}=\rho r \omega^{2}
$$

The flow problem is subjected to the following conditions:

$$
\begin{gathered}
V_{r}=0, V_{\varphi}=0, V_{z}=-V, D_{\alpha} \frac{\partial a}{\partial z}=k_{s} a, D_{\beta} \frac{\partial b}{\partial z}=-k_{s} a \text { at } z=0, \\
V_{r} \rightarrow 0, V_{\varphi} \rightarrow r \omega, a \rightarrow a_{0}, b \rightarrow 0 \text { as } z \rightarrow \infty .
\end{gathered}
$$

Using von-Kármán variables together with new functions $\phi(\zeta)$ and $h(\zeta)$ :

$$
\left(V_{r}, V_{\varphi}, V_{z}\right)=(r \omega F(\zeta), r \omega G(\zeta), \sqrt{v \omega} H(\zeta)),(a, b)=\left(a_{0} \phi(\zeta), a_{0} h(\zeta)\right),
$$

where $\zeta=z(\omega / v)^{1 / 2}$ is the similarity variable. Making use of the standard boundary layer approximations and then using Equation (11), Equations (3)-(8) convert into the following ODEs:

$$
\begin{gathered}
H^{\prime}+2 F=0, \\
F^{\prime \prime}-F^{2}-F^{\prime} H+G^{2}-1=0, \\
G^{\prime \prime}-2 F G-G^{\prime} H=0, \\
\frac{1}{S c} \phi^{\prime \prime}-H \phi^{\prime}-K \phi h^{2}=0, \\
\frac{\delta}{S c} h^{\prime \prime}-H h^{\prime}+K \phi h^{2}=0 .
\end{gathered}
$$


The boundary conditions are transformed as:

$$
\begin{gathered}
F(0)=G(0)=0, H(0)=-A, \phi^{\prime}(0)=K_{s} \phi(0), \delta h^{\prime}(0)=-K_{s} \phi(0) \text { at } z=0, \\
F \rightarrow 0, G \rightarrow 1, \phi \rightarrow 1, h \rightarrow 0 \text { as } z \rightarrow \infty .
\end{gathered}
$$

where $S c$ denotes the Schmidt number, $A$ is the suction-strength parameter, $\delta$ is the ratio of diffusion coefficients, $K$ measures the strength of homogeneous reaction, and $K_{s}$ is the heterogeneous-reaction strength parameter. These are defined as follows:

$$
S c=\frac{v}{D_{\alpha}}, \operatorname{Pr}=\frac{\mu c_{p}}{\kappa}, A=\frac{V}{\sqrt{v \omega}}, \delta=\frac{D_{\beta}}{D_{\alpha}}, K=\frac{k_{c} a_{0}^{2}}{\omega}, K_{s}=\frac{k_{s}}{D_{\alpha}} \sqrt{\frac{\nu}{\omega}} .
$$

Author et al. [15] stated that the diffusion coefficients of species $\alpha$ and $\beta$ are comparable in size in many applications. This requires $D_{\alpha}$ and $D_{\beta}$ to be equal or equivalently $\delta \approx 1$. Considering this assumption and the boundary conditions in Equation (17), we can express:

$$
\phi(\zeta)+h(\zeta)=1
$$

which allows us to combine Equations (15) and (16) into single equation:

$$
\frac{1}{S c} \phi^{\prime \prime}-H \phi^{\prime}-K \phi(1-\phi)^{2}=0,
$$

subject to the conditions:

$$
\phi^{\prime}(0)=K_{s} \phi(0) \text { and } \phi(\infty) \rightarrow 1
$$

\subsection{Asymptotic Behavior}

For large $\zeta$, we can assume that:

$$
\begin{gathered}
H(\zeta)=H_{0}+\widetilde{H}(\zeta), \\
\phi(\zeta)=1+\widetilde{\phi}(\zeta),
\end{gathered}
$$

where $H_{0}$ is a constant and $\widetilde{H}$ and $\widetilde{\phi}$ are small. Substituting Equations (22) and (23) into Equation (20) and then linearizing produces:

$$
\frac{1}{S c} \widetilde{\phi^{\prime \prime}}-H_{0} \widetilde{\phi^{\prime}}=0
$$

Integrating Equation (24) twice, we have:

$$
\widetilde{\phi}=-\frac{c_{1}}{H_{0} S c}+c_{2} \exp \left(H_{0} S c \zeta\right)
$$

where $c_{1}$ and $c_{2}$ are arbitrary constants. Since $\widetilde{\phi}$ vanishes at infinity, we choose $c_{1}=0$. Equation (25) reflects the exponential decay in the solution, or the existence of a boundary layer on the disk, which is possible only when $H_{0}<0$.

\subsection{Exact Solution in Case of Large Suction When $K=0$}

We integrate concentration Equation (20) twice and then use the boundary conditions in Equation (21) to find $\phi(\zeta)$ :

$$
\phi(\zeta)=\frac{1}{K_{s} I(\infty)+1}\left[1+K_{s} I(\zeta)\right]
$$


where the integral $I(\zeta)$ is of the form:

$$
I(\zeta)=\int_{0}^{\zeta} \exp \left(S c \int_{0}^{\zeta} H(\lambda) d \lambda\right) d \zeta
$$
the form:

Since $I^{\prime}(\zeta)=\exp \left(S c \int_{0}^{\zeta} H(\lambda) d \lambda\right)$ and $I^{\prime}(0)=1$, the concentration gradient at the surface assumes

$$
\phi^{\prime}(0)=\frac{K_{s}}{K_{s} I(\infty)+1} I^{\prime}(0)=\frac{K_{s}}{K_{s} I(\infty)+1} .
$$

For a sufficiently large $A$, the axial velocity becomes constant, $H=H_{0}$. The integral $I(\zeta)$ in Equation (27) can be simplified to the form:

$$
I(\zeta)=\frac{1}{S c H_{0}}\left(\exp \left(S_{c H} H_{0} \zeta\right)-1\right)
$$

The above expression produces $I(\infty)=-1 / S c H_{0}$, provided $H_{0}<0$. Hence, Equation (28) becomes:

$$
\phi^{\prime}(0)=\frac{S c H_{0} K_{s}}{S c H_{0}-K_{s}} .
$$

The practically significant quantity is the skin friction factor experienced at the disk, defined as:

$$
C_{f}=\frac{\sqrt{\tau_{r}^{2}+\tau_{\varphi}^{2}}}{\rho(r \omega)^{2}}
$$

where $\tau_{r}$ and $\tau_{\varphi}$ denote the radial and circumferential wall stresses, respectively. Using Equation (11), Equation (31) is reducible to the form:

$$
C_{f}\left(\frac{r^{2} \omega}{v}\right)^{\frac{1}{2}}=\sqrt{F^{\prime}(0)^{2}+G^{\prime}(0)^{2}} .
$$

The total amount of fluid swept radially from a cylinder of radius $R$ taken along the z-axis is:

$$
Q=2 \pi R \int_{0}^{\infty} V_{r} d z=2 \pi R^{2} \sqrt{v \omega} \int_{0}^{\infty} F d \zeta=-\pi R^{2} \sqrt{v \omega}[H(\infty)+A]
$$

which reveals that volume flow is connected with the entrainment velocity $H(\infty)$ and suction-strength parameter $A$.

\section{Numerical Results and Discussion}

The influence of homogeneous-heterogeneous reactions on the Bödewadt flow with suction was the main focus of this research. Following Rahman and Andersson [12], we relied on MATLAB's (MathWorks, Massachusetts, USA) routine bvp4c to estimate velocity, temperature, and concentration distributions above the disk. During the computational procedure, a small value of $\zeta_{\infty}$ was and then it was gradually increased while observing the residuals of $F, G$, and $\phi$ at infinity so that they remained less than $10^{-6}$. The value of $\zeta_{\infty}=20$ appears sufficient to meet the specified tolerance criterion.

Like the thermal energy equation, the mass transfer problem in Bödewadt flow can exhibit a physically compatible solution only when a wall permeability condition is imposed. A comparison of the results for $F^{\prime}(0)$ and $G^{\prime}(0)$ with those estimated by [12], and such comparison seems convincing (Table 1). The asymptotic approximation of $\phi$ (in Equation (30)) agrees well with the computational results for larger values of $A$ (Table 2). Table 3 includes the numerical normalized skin friction data, far 
field axial velocity, and wall concentration gradient. Wall transpiration provides increases the skin friction encountered at the disk, flow rate, and species concentration at the disk. However, the suction effects contribute largely toward improvement in the wall cooling rate, which is vital for many technical processes. Table 4 shows that wall concentration increases with increasing values of Sc. This is in compliance with higher values of $S c$ indicating relatively lower mass diffusion, thereby resulting in a thinner concentration boundary layer and higher wall concentration gradient. Increasing either $K$ or $K_{s}$ results in more consumption of the reactants, thereby lowering surface concentration.

Figure $1 \mathrm{a}, \mathrm{b}$ show the evolution of velocity curves $(H, F$, and $G)$ with and without the suction effect. The axial velocity remains constant at a certain distance above the disk, while its magnitude is reduced near the disk due to viscous drag. For the conservation principle to hold, the axial flow remains upward in pure Bödewadt flow. Velocity components have oscillatory profiles with amplitude inversely proportional to the axial distance. Note that the axial velocity at infinity becomes negative when sufficiently high suction velocity is imposed.

Figure 2a-d shows the distribution of velocity components $(H, F$, and $G)$ and concentration profile $(\phi)$ for a variety of suction strength parameters. The amplitude of oscillations considerably decreases when boundary suction is introduced in the model. The strength of the axial flow grows with increasing values of $A$. However, the inward radial flow induced by the pressure gradient slows with increasing $A$. The circumferential flow also decelerates with the inclusion of wall permeability. Figure 2a shows that the axial velocity profile $H$ reaches some constant value $H_{0}$ when a sufficiently high value of $A$ is considered. The results in Figure 2a reveal that a higher volume of fluid is drawn toward the disk when a larger $A$ is used. The asymptotic solution in Equation (25) shows that a solution for concentration would be possible only when $H(\infty)$ is negative. For this reason, $\phi$ has a physically inconsistent solution when $A=0$, in which $H(\infty)$ has a positive value (Figure 1a). However, for $A=1$ where $H(\infty)$ is negative (Figure $1 b$ ), a physically consistent solution for $\phi$ is accomplished. In conclusion, the axially upward flow is a result of an unphysical solution to the advection-diffusion problem. This physical inconsistency can be overcome by reversing the direction of the axial flow via the application of sufficiently high suction velocity. Therefore, the damping effect, arising as a consequence of wall suction, can overcome the instabilities in Bödewadt flow known from the published literature.
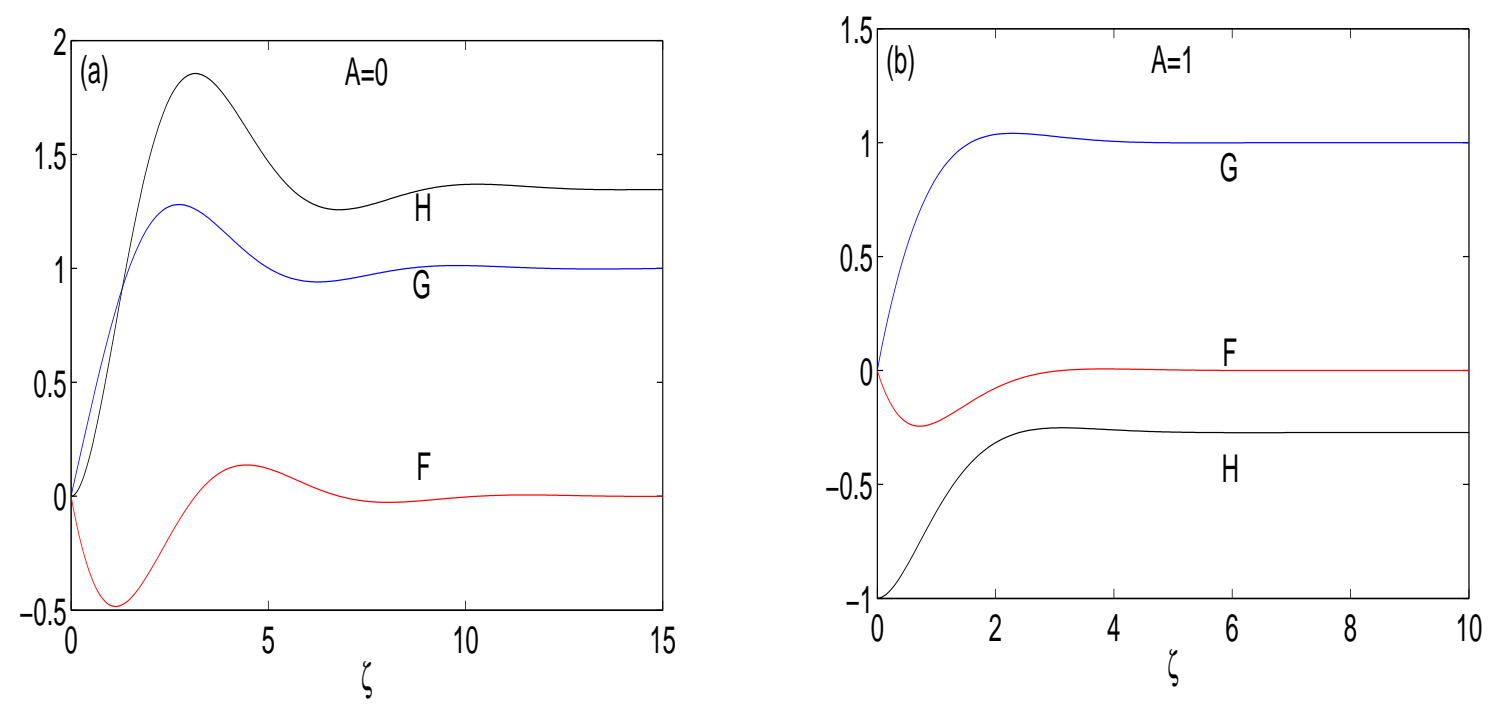

Figure 1. Velocity components $(H, F$, and $G$ with and without suction effects. 

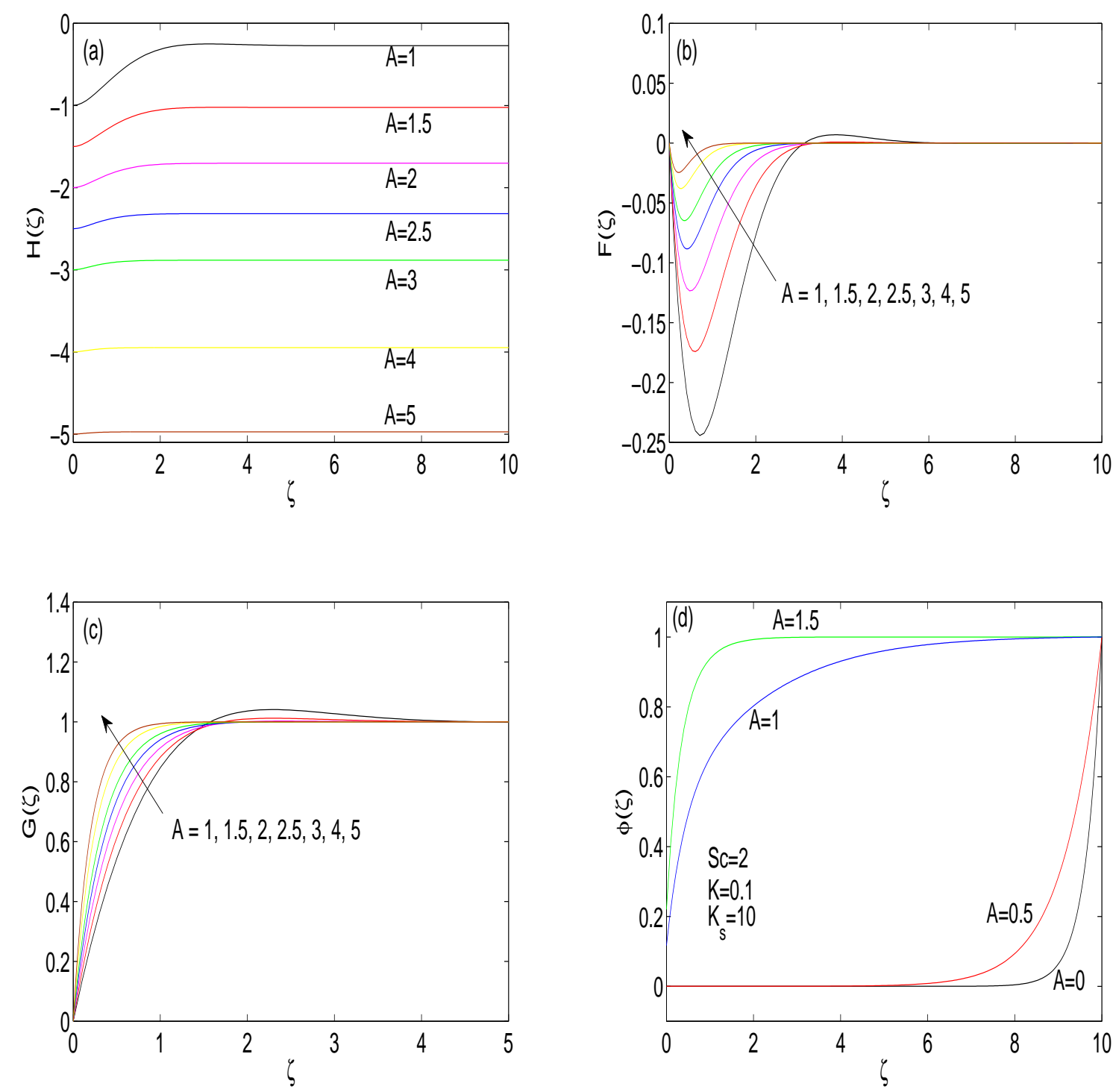

Figure 2. Velocity components $(H, F$, and $G)$ and $\phi$ for different values of $A$.

We determined the evolution of concentration profile $\phi$ at different values of involved parameters, as shown in Figure 3a-d. Graphs of $\phi$ against $\zeta$ for various values of suction-strength parameters are depicted in Figure $3 a$. The concentration distribution has no meaningful solution in the absence of suction, an outcome that has been briefly explained in previous reports. Hence, we pursued the numerical solution of $\phi$ for $A \geq 1$. The general trend in the concentration profile a constant value at the wall and its value grows monotonically with increasing axial distance. The concentration boundary layer does not exist when $K_{s}=0$, which is also apparent from the condition in Equation (21). The concentration boundary layer appears to decrease when $A$ increases. Also, the profile of $\phi$ is broader at $A=1$ than at $A=3$. Figure $3 b$ portrays the plots of $\phi$ as the Schmidt number $S c$ changes. $\phi$ decreases sharply for increasing values of Sc. Physically, for a higher Schmidt number, $S c=v / D_{\alpha}$, mass diffusion coefficient is very small compared with the momentum diffusion. To understand the onset of homogeneous reaction parameter $K$, Figure $3 \mathrm{c}$ was plotted. The curves of $\phi$ flatten as parameter $K$ increases. Physically, by increasing either $K$ or $K_{s}$, more reactants are consumed in the boundary layer, thereby decreasing $\phi(\zeta)$. 

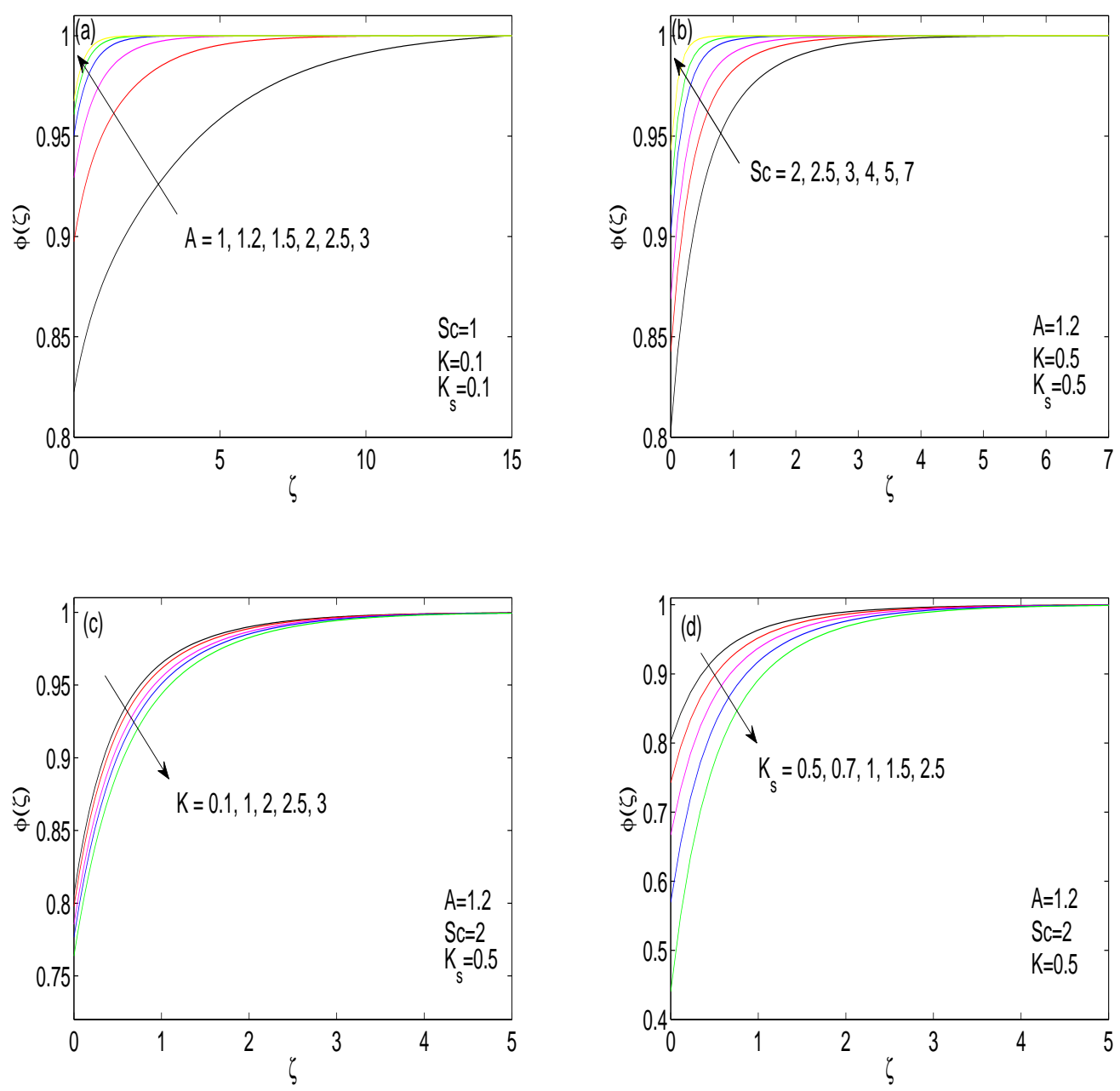

Figure 3. Concentration profile $(\phi)$ for different values of embedded parameters.

The profiles of surface concentration $\phi(0)$ are presented in Figure $4 a, b$. A linear decay occurs in the surface concentration when either $K$ or $K_{s}$ becomes large. Hence, for a fixed $K_{s}$, the wall concentration gradient $\phi^{\prime}(0)$ vanishes for sufficiently high values of $K$. Physically, the chemical reaction occurs at some distance high above the disk when $K$ is sufficiently large. However, Figure $4 \mathrm{~b}$ shows that $\phi(0)$ grows non-linearly as Sc increases.
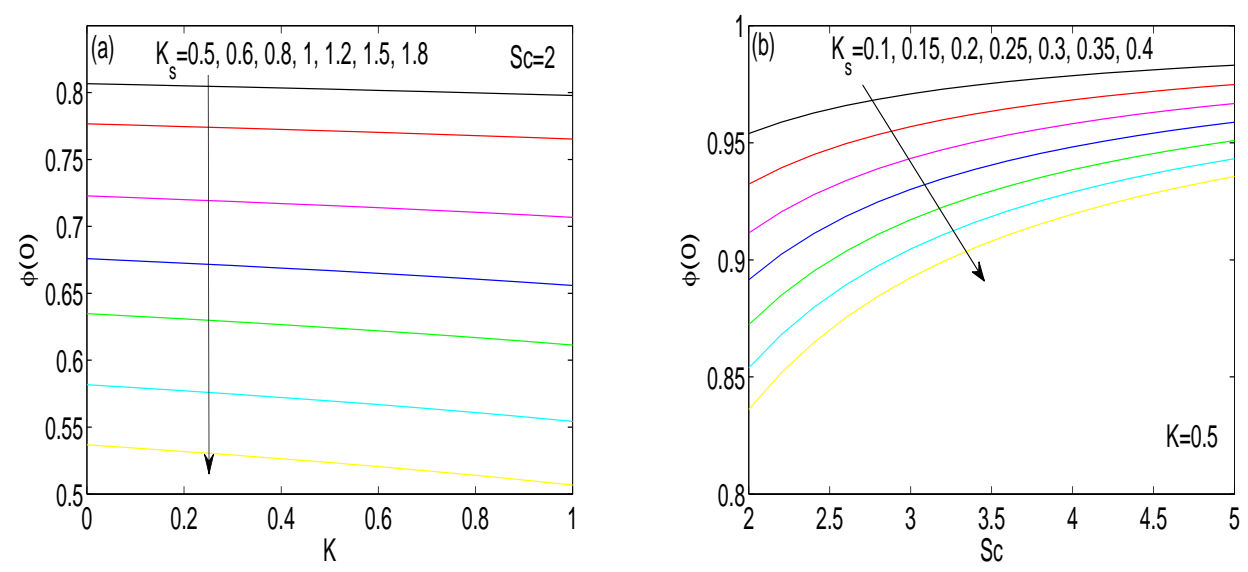

Figure 4. Graph of surface concentration $(\phi(0))$ versus (a) $K$ and (b) $S c$ at different values of heterogeneous reaction parameter $K_{s}$. 
Table 1. Comparison of values of $F^{\prime}(0)$ and $G^{\prime}(0)$ obtained by Rahman and Andersson [12] for various suction-strength parameters.

\begin{tabular}{ccccc}
\hline \multirow{2}{*}{$A$} & \multicolumn{2}{c}{$-F^{\prime}(0)$} & \multicolumn{2}{c}{$G^{\prime}(0)$} \\
\cline { 2 - 5 } & Present & {$[12]$} & Present & [12] \\
\hline 0 & 0.9419 & 0.9420 & 0.7728 & 0.7729 \\
\hline 0.5 & 0.9033 & 0.9033 & 1.0650 & 1.0652 \\
\hline 1 & 0.8351 & 0.8351 & 1.3883 & 1.3885 \\
\hline 2 & 0.6468 & 0.6468 & 2.1560 & 2.1560 \\
\hline 3 & 0.4800 & 0.4800 & 3.0611 & 3.0611 \\
\hline
\end{tabular}

Table 2. Comparison of numerical results for $\phi^{\prime}(0)$ and analytical Equation (30) for a variety of suction-strength parameters when $S_{c}=5$ and $K_{s}=0.5$.

\begin{tabular}{cccc}
\hline \multirow{2}{*}{$A$} & \multicolumn{3}{c}{$\phi^{\prime}$ (0) } \\
\cline { 2 - 4 } & Numerical & Analytical Equation (30) & Difference \\
\hline 1 & 0.451842 & 0.454545 & 0.002703 \\
\hline 2 & 0.476083 & 0.476190 & 0.000107 \\
\hline 3 & 0.483855 & 0.487871 & 0.000016 \\
\hline 4 & 0.487801 & 0.487805 & 0.000004 \\
\hline 5 & 0.490195 & 0.490196 & 0.000001 \\
\hline 6 & 0.491803 & 0.491803 & 0.000000 \\
\hline
\end{tabular}

Table 3. Values of wall skin friction, volumetric flow rate, wall concentration, and wall concentration gradient for different values of $A$ when $K=0.5=K_{S}$ and $S c=2$.

\begin{tabular}{ccccc}
\hline$A$ & $\sqrt{F^{\prime}(0)^{2}+G^{\prime}(0)^{2}}$ & $H(\infty)$ & $\phi(0)$ & $\phi^{\prime}(0)$ \\
\hline 1 & 1.312385 & -0.268272 & 0.7205807 & 0.360290 \\
\hline 1.5 & 1.810597 & -1.023830 & 0.848774 & 0.424387 \\
\hline 2 & 2.533371 & -1.703410 & 0.886562 & 0.443281 \\
\hline 2.5 & 3.525320 & -2.316386 & 0.908228 & 0.454114 \\
\hline 3 & 4.800394 & -2.883492 & 0.922698 & 0.461349 \\
\hline 3.5 & 6.354416 & -3.423185 & 0.933146 & 0.466573 \\
\hline 5 & 12.616804 & -4.972426 & 0.952344 & 0.476172 \\
\hline 6 & 18.081658 & -5.983917 & 0.959984 & 0.479992 \\
\hline 10 & 50.0295589 & -9.996503 & 0.975608 & 0.487804 \\
\hline
\end{tabular}

Table 4. Computation results of wall concentration $\phi(0)$ and wall concentration gradient $\phi^{\prime}(0)$ for various values of $K, K_{s}$, and $S c$ when $A=1.2$.

\begin{tabular}{ccccc}
\hline$S \boldsymbol{c}$ & $\boldsymbol{K}$ & $\boldsymbol{K}_{\boldsymbol{s}}$ & $\phi(0)$ & $\phi^{\prime}(0)$ \\
\hline 0.5 & 0.5 & 0.5 & 0.432011 & 0.216005 \\
\hline 1 & & 0.618288 & 0.309144 \\
\hline 2 & & 0.802841 & 0.401421 \\
\hline 5 & & 0.920734 & 0.460367 \\
\hline 10 & & 0.959663 & 0.479832 \\
\hline 2 & & 0.806127 & 0.403064 \\
\hline & 0.1 & 0.798183 & 0.399091 \\
\hline & 1 & 0.786050 & 0.393025 \\
\hline & & 0.765414 & 0.382707 \\
\hline & 3.5 & 0.742473 & 0.371236 \\
\hline & 0.5 & 0.802841 & 0.401421 \\
\hline & 0.5 & 0.667402 & 0.667402 \\
\hline & 1 & 0.497335 & 0.994669 \\
\hline & 2 & 0.281515 & 1.407573 \\
\hline & 5 & 0.163605 & 1.636049 \\
\hline & 10 & &
\end{tabular}




\section{Concluding Remarks}

The onset of homogeneous-heterogeneous reactions in Bödewadt flow around a permeable disk was considered in this study. We showed that the governing problem can be reduced to a self-similar system. Numerical calculations were presented for velocity and concentration distributions above the disk. Notable outcomes of this research are summarized below:

(1) A meaningful solution for concentration $\phi$ exists only when a permeable disk with sufficiently high suction is considered. This is also apparent from the asymptotic expression of $\phi$ valid for large axial distance $\zeta$.

(2) Suction tends to suppress velocity and concentration profiles. These, in turn, elevate the concentration of chemical species at the disk.

(3) In large suction case with $K=0$, a closed form solution is obtained that supports the numerical solution obtained.

(4) By increasing either or $K_{s}$, the consumption of reactants within the boundary layer increases, thereby decreasing concentration.

(5) The wall concentration gradient asymptotically vanishes when the rate of chemical reaction increases.

Author Contributions: T.R. developed the flow model. Both T.R. and M.M.H. performed computational analysis and wrote the paper.

Funding: No specific funding was received for this work.

Conflicts of Interest: The authors declare that there is no conflict of interest.

\section{References}

1. Von-Kármán, T. Uber laminare and turbulente Reibung. Z. Angew. Math. Mech. 1921, 1, 233-252.

2. Bödewadt, U.T. Die Dreh stromunguber festem Grund. Z. Angew. Math. Mech. 1940, 20, 241-253. [CrossRef]

3. Batchelor, G.K. Note on the class of solutions of the Navier-Stokes equations representing steady non-rotationally symmetric flow, Quart. J. Appl. Math. 1951, 4, $29-41$.

4. Rogers, M.H.; Lance, G.N. The rotationally symmetric flow of a viscous fluid in the presence of an infinite rotating disk. J. Fluid Mech. 1960, 7, 617-631. [CrossRef]

5. Ackroyd, J.A.D. On steady flow produced by a rotating dick with either suction or injection. J. Eng. Math. 1978, 12, 207-220. [CrossRef]

6. Watson, L.T.; Wang, C.Y. Deceleration of a rotating disk in a viscous fluid. Phys. Fluids 1979, 22, 2267-2269. [CrossRef]

7. Fransaer, J.; Roos, J.R.; Cellis, J.P. Fluid Flow past a rotating disk. Chem. Eng. Sci. 1991, 46, 1194-1196. [CrossRef]

8. Sahoo, B. Effects of slip on steady Bödewadt flow and heat transfer of an electrically conducting non-Newtonian fluid. Commun. Nonlinear Sci. Num. Simul. 2011, 16, 4284-4295. [CrossRef]

9. Sahoo, B.; Gorder, R.A.V.; Andersson, H.I. Steady revolving flow and heat transfer of a non-Newtonian Reiner-Rivlin fluid. Int. Commun. Heat Mass Transf. 2012, 39, 336-342. [CrossRef]

10. Turkyilmazoglu, M. Bödewadt flow and heat transfer over a stretching stationary disk. Int. J. Mech. Sci. 2015, 90, 246-250. [CrossRef]

11. Mustafa, M.; Khan, J.A.; Hayat, T.; Alsaedi, A. On Bödewadt flow and heat transfer of nanofluids over a stretching stationary disk. J. Mol. Liq. 2015, 211, 119-125. [CrossRef]

12. Rahman, M.; Andersson, H.I. On heat transfer in Bödewadt flow. Int. J. Heat Mass Transf. 2017, 112, 1057-1061. [CrossRef]

13. Khan, J.A.; Mustafa, M.; Hayat, T.; Alzahrani, F. Numerical study for Bödewadt flow of water based nanofluid over a deformable disk: Buongiorno model. Ind. J. Phys. 2017, 91, 527-533. [CrossRef]

14. Mustafa, M.; Pop, I.; Naganthran, K.; Nazar, R. Entropy generation analysis for radiative heat transfer to Bödewadt slip flow subject to strong suction-strength. Eur. J. Mech. B/Fluids 2018, 72, 179-188. [CrossRef] 
15. Chaudhary, M.A.; Merkin, J.H. A simple isothermal model for homogeneous-heterogeneous reactions in boundary layer flow. I Equal diffusivities. Fluid Dyn. Res. 1995, 16, 311-333. [CrossRef]

16. Merkin, J.H. A model for isothermal homogenous-heterogeneous reaction in boundary-layer flow. Math. Comput. Model 1996, 24, 125-136. [CrossRef]

17. Bachok, N.; Ishak, A.; Pop, I. On the stagnation-point flow towards a stretching sheet with homogeneousheterogeneous reactions effects. Commun. Nonlinear Sci. Numer. Simul. 2011, 16, 4296-4302. [CrossRef]

18. Khan, W.A.; Pop, I. Effects of homogeneous-heterogeneous reaction on the viscoelastic fluid towards a stretching sheet. ASME J. Heat Transf. 2012, 134, 064506. [CrossRef]

19. Hayat, T.; Imtiaz, M.; Almezal, S. Modeling and analysis for three-dimensional flow with homogeneousheterogeneous reactions. AIP Adv. 2015, 5, 107209. [CrossRef]

20. Hayat, T.; Rashid, M.; Imtiaz, M.; Alsaedi, A. Nanofluid flow due to rotating disk with variable thickness and homogeneous-heterogeneous reactions. Int. J. Heat Mass Transf. 2017, 113, 96-105. [CrossRef]

21. Maqsood, N.; Mustafa, M.; Khan, J.A. Numerical tackling for viscoelastic fluid flow in rotating frame considering homogeneous-heterogeneous reaction. Result Phys. 2017, 7, 3475-3481. [CrossRef]

22. Khan, M.I.; Waqas, M.; Hayat, T.; Alsaedi, A. A comparative study of Casson fluid with homogeneousheterogeneous reactions. J. Colloid Interf. 2017, 498, 85-90. [CrossRef]

23. Khan, M.I.; Hayat, T.; Waqas, M.; Alsaedi, A. Outcome for chemically reactive aspect in flow of tangent hyperbolic material. J. Mol. Liq. 2017, 230, 143-151. [CrossRef]

24. Khan, M.I.; Hayat, T.; Waqas, M.; Alsaedi, A. Impact of heat generation/absorption and homogeneousheterogeneous reactions on flow of Maxwell fluid. J. Mol. Liq. 2017, 233, 465-470. [CrossRef]

25. Xu, N.L.; Xu, H.; Raees, A. Homogeneous-heterogeneous reactions in flow of nanofluids near the stagnation region of a plane surface: The Buongiorno's model. Int. J. Heat Mass Transf. 2018, 125, 604-609. [CrossRef]

26. Mahdy, A. Impacts of homogeneous-heterogeneous chemical reactions and inclined magnetic field on unsteady nanofluids flow. AIP Adv. 2018, 8, 115109. [CrossRef]

(C) 2019 by the authors. Licensee MDPI, Basel, Switzerland. This article is an open access article distributed under the terms and conditions of the Creative Commons Attribution (CC BY) license (http://creativecommons.org/licenses/by/4.0/). 